\title{
Correlation of Haplotypes of a Fungal Plant Pathogen with their Respective Host Species of Origin
}

\author{
Stephanie S. Garr, Carolyn Hughes, Jacqueline Welch, Scott A. Brown, and Michael H. Perlin, Department of \\ Biology, University of Louisville, Louisville, KY 40292
}

\begin{abstract}
Garr, S. S., Hughes, C., Welch, J., Brown, S. A., and Perlin, M. H. 1997. Correlation of haplotypes of a fungal plant pathogen with their respective host species of origin. Plant Dis. 81:936941.

The anther smut fungus, Microbotryum violaceum, infects over 200 species of Caryophyllaceae (Pinks). However, limited published studies, as well as anecdotal evidence, suggest that each isolate of the fungus is restricted to one or a few species that it can productively infect. In the absence of physical differences, it would be useful to have molecular markers to identify individuals with specific host ranges prior to genetic analyses of host preference. With this purpose in mind, 17 isolates from eight different host species were characterized for differences in their respective $\gamma$-tubulin genes. The region of the gene including the sixth and seventh introns and some surrounding coding regions was amplified and sequenced and the results were analyzed phylogenetically. Despite the small sample size and the geographical distribution of their respective host plants, isolates from the same host species showed no differences in the DNA regions examined; isolates of closely related pathovars also grouped together. In contrast, relative to the corresponding regions from other pathovars, isolates from host species that were genetically or taxonomically more distant showed a marked number of differences in both introns and in the third (wobble) position of codons in the seventh exon. Thus, DNA sequence differences in this highly conserved gene may be used to distinguish isolates from different host species. Such information may prove useful as markers for the different formae speciales in future analyses of host preference.
\end{abstract}

Additional keywords: intron polymorphisms, pathogenic fungi, pathovars

It is likely that the life histories of a pathogen and its host(s) will be tightly coupled and may coevolve $(2,25)$. Because the availability of host stages susceptible to infection may be limited by space and time, the pathogen must have features of its own life history that will allow its persistence in a host population. Add to this requirement the need for compatibility between pathogen and host that results from specific biochemical-physiologicalmorphological cues and a complex picture of host-pathogen interaction begins to emerge.

The goal of this study was to identify relationships among different isolates of a fungal plant pathogen. Microbotryum violaceum (Pers.:Pers.) Deml \& Oberw. (7) (Ustilago violacea [Pers.] Roussel) is a heterobasidiomycete fungus that produces its spores in the anthers of the plants it infects. Over 200 species of Caryophyllaceae (Pinks) serve as host of $M$. violaceum, although individual isolates

Corresponding author: M. H. Perlin

E-mail: mhper101@ULKYVM.Louisville.Edu

Accepted for publication 12 May 1997.

Publication no. D-1997-0603-01R

(C) 1997 The American Phytopathological Society appear to be limited in host range to one or a few species $(32,33)$. This relationship between host and pathogen would thus seem to define pathovars or formae speciales of the fungus (33). However, given that completion of the $M$. violaceum life cycle occurs only in an appropriate host plant, the task of conducting a complete host range analysis for each isolate could prove quite daunting. At least one molecular genetic approach has demonstrated relationships among sporidial isolates from the same host species (23). Electrophoretic karyotypes of strains produced by pulsefield gel electrophoresis revealed similarities among strains from the same host and, in some cases, clear differences among different formae speciales.

Introns, the transcribed regions of genes that are spliced out of the mature message before translation, are found in many eukaryotic genes. Because most introns lack information for protein coding, with the exception of sequences required for proper splicing, there should be few selective constraints on mutations in these regions. As such, intron sequences might be expected to be useful for intraspecific or population studies. Polymorphisms in introns have been used to distinguish different breeds of cows (14), as markers for human disease $(15,18,19,22)$, to help clarify hidden patterns of structure in natural populations of humpback whale (21), and to distinguish varieties of a fungal species (30). Here we examine relatedness among formae speciales of $M$. violaceum using phylogenies of two introns and one exon in the $\gamma$-tubulin-encoding gene of the fungus.

\section{MATERIALS AND METHODS}

Fungal strains. $M$. violaceum is a dimorphic fungus; haploid sporidial cells of opposite mating type are produced via meiosis of diploid teliospores from anthers after germination of such spores on appropriate surfaces, e.g., plant tissue or on agar plates. Sporidia reproduce by budding and may be propagated in the laboratory on artificial media (12). Although the mating process between cells of opposite mating type can also be demonstrated on artificial media, stable differentiation and completion of the life cycle only occur in planta. A list of $M$. violaceum sporidial strains used and the hosts from which they were isolated is found in Table 1.

Target gene amplification and nucleotide sequencing. The region of the $M$. violaceum $\gamma$-tubulin-encoding gene (EMBL Accession No. X68132) between positions 1319 and 2054 was amplified for each isolate via polymerase chain reaction (PCR). This region included the sixth and seventh introns as well as the seventh exon of the gene. The amplified region also contained additional surrounding squences not included in the analyses for this study because DNA sequence data were not obtained for these regions for all isolates. The primers for amplification were designed from positions 1319 to 1340 (primer T1) and from positions 2054 to 2031 (T2) of the EMBL sequence using the software AGCT PCR primer analysis package (AGCT, Irvine, CA) and were: T1 (5'GGTTTTATGCTCCTCCATTCCA-3') and T2 (5'-GATGTTGAGGATTGAGATGTAGCA-3'). Isolation of genomic DNA from sporidia and reaction conditions for PCR were as described previously (17). Direct determination of DNA sequence from PCR products employed a kit (Amersham, Arlington Heights, IL) in which treatment with exonuclease I and shrimp alkaline phosphatase was followed by chain termination reactions (24) using T7 DNA polymerase. Reaction products were radiolabeled with $\left[\alpha{ }^{35} \mathrm{~S}\right]$-dATP $(1,000 \mathrm{Ci} / \mathrm{mmol}$, Amersham) in the reactions and were separated in $6 \%$ acrylamide gels containing a 
glycerol-tolerant buffer system (Amersham). Dried gels were exposed to Kodak Biomax MR X-ray film for autoradiography.

Phylogenetic analyses. Sequences from each isolate for the sixth and seventh introns, the seventh exon, and all three regions combined were entered and saved as separate MSG files for the GCG, version 8 (Genetics Computer Group, Madison, WI) software package. They were aligned using Clustal W (31), and then analyzed using MEGA 1.01 (Pennsylvania State University, University Park, PA) or PAUP 3.1.1 (Illinois Natural History Survey, Champaign, IL). Distance parameters/methods used in MEGA were: number of differences (transitions and transversions or transversions only), method of Jukes and Cantor (13), or the method of Tamura and Nei (29). Phylogenetic analyses using the unweighted pair-group method (UPGMA) or neighbor-joining (NJ) used completedeletion or pairwise deletion to produce the trees. Bootstrap analysis of branch-andbound trees was done with MEGA for 500 replications for maximum likelihood (ML) or $\mathrm{NJ}$ trees or for 100 replications for maximum parsimony analysis (MP) trees using PAUP 3.1.1.

\section{RESULTS}

MP was used to construct phylogenies of different forma specialis isolates of $M$. violaceum based on $\gamma$-tubulin-encoding gene sequences of the isolates. Due to a number of insertions in the sixth and seventh intron, the largest combined sequence alignment included a total of 334 nucleotides (Fig. 1). During the alignments by Clustal W, gaps were introduced for the 17 different taxa to facilitate the alignment. Of 334 alignable sites in the combined sequence for all three regions, 90 were variable and phylogenetically informative and were used in MP analyses. A single most parsimonious tree was found with a length of 108 and a consistency index of 0.926. Bootstrap analysis of branch-and- bound trees for 100 replications yielded the tree shown in Figure 2.

Additional analyses were also carried out via ML using UPGMA and distance comparisons were made using NJ. The topology of all trees for a given region was consistent whether MP, ML, or NJ were used. As predicted above, character changes observed between different isolates appeared to have preserved expected splice signals in both introns and occurred only in synonymous positions in the codons for the seventh exon. Furthermore, absolute numbers of differences between isolates were higher for either intron than for the seventh exon. In general, isolates from the same host species grouped together in all analyses: i.e., all isolates from Silene latifolia formed a clade, as did isolates from $S$. acaulis, $S$. virginica, Melandrium rubrum, and Dianthus carthusianorum. Also, in some instances, isolates from similar host species grouped together: An isolate from an S. latifolia/S. dioica hybrid host and an isolate from $S$. dioica consistently grouped with the isolates from $S$. latifolia and could not be distinguished from these latter isolates based on analyses of the sequences in this study.

In trees for intron 6 alone, the isolates from $M$. rubrum were included with the $S$. latifolia clade. In all other trees, the $M$. rubrum isolates formed a sister group to the isolates from $S$. latifolia. For intron 6, the $S$. acaulis clade was always the most distant from the $S$. latifolia clade, followed by the $S$. virginica and $D$. carthusianorum clades, respectively. In all analyses of exon 7 the D. carthusianorum isolates were most distant from the others, and the $S$. virginica and $S$. acaulis clades were sister groups. Analyses of intron 7 also found the $S$. virginica and $S$. acaulis clades to be sister groups of each other. When only transversions were considered, the $S$. acaulis clade was closer to the S. latifolia/M. rubrum group.
Trees for the combined three regions (i.e., intron 6, exon 7, and intron 7) consistently placed the $M$. rubrum isolates as a sister group to the $S$. latifolia clade, while UWO11-1, the lone isolate from L. floscuculi, formed a basal group to these strains. All of these analyses found the $S$. virginica isolates to be most distant from the remaining isolates.

Pairwise estimates of sequence divergence were obtained using either the formula of Jukes and Cantor (13), that of Tamura and Nei (29), or simply as number of differences. These estimates ranged from 0.009 ( $S$. latifolia isolates clade vs. $M$. rubrum isolates) to 0.198 (S. acaulis isolates vs. $S$. virginica isolates) and were used to construct an $\mathrm{NJ}$ tree (data not shown). A similar tree was also produced using UPGMA and complete-deletion estimates to produce an ML tree, which was subjected to bootstrap analysis (not shown). The groups defined in that tree were essentially those defined in the majority rule $(>50 \%)$ bootstrap MP analysis.

\section{DISCUSSION}

The anther smuts are systemic fungi for which free-living saprophytic stages are relatively unimportant and for which transmission via seed is unlikely (28). These fungi specialize in forming sori in the anthers of hosts in the Caryophyllaceae, Dipsacaceae, Liliaceae, and Portulacaceae. The population biology and genetics of $M$. violaceum, which infects members of the Caryophyllaceae, have been examined extensively $(1,12) . M$. violaceum is primarily transmitted between different adult plants by insects which serve both as pollinators and as vectors for the pathogen (1). Given such limitations, transmission of the fungus between different host species may be restricted. In fact, although diseased populations of S. latifolia and $S$. virginica are known to occur in close proximity, as evidenced by allozyme profiles, there seems to have been little or

Table 1. List of Mycobotryum violaceum strains

\begin{tabular}{|c|c|c|c|c|c|}
\hline Strain & Characteristics & Host & Pathovar & Location & Source $^{\mathrm{z}}$ \\
\hline $1 . \mathrm{C} 436 y$ & $a_{1} y$ & Silene dioica and S. latifolia & & Scotland & $\mathrm{a}$ \\
\hline 2.8 .11 .81 & $\mathrm{a}_{2} w$ met $\mathrm{Bot} 400$ & S. latifolia & & England & $\mathrm{a}$ \\
\hline SIX & $a_{1}$ & S. latifolia & & Virginia (USA) & $\mathrm{b}$ \\
\hline NINE & $a_{2}$ & S. latifolia & & Virginia (USA) & $\mathrm{b}$ \\
\hline 22000 & $a_{1}$ & S. latifolia & & England & $\mathrm{c}$ \\
\hline 22001 & $a_{2}$ & S. latifolia & & England & $\mathrm{c}$ \\
\hline UWO2 & $\begin{array}{l}a_{2} \\
a_{2}\end{array}$ & S. latifolia/S. dioica hybrid & SA/D-1 & England & $\mathrm{c}$ \\
\hline UWO14 & $a_{1}$ & S. dioica & SD8 & Scotland & $\mathrm{c}$ \\
\hline UWO11-1 & $a_{1}$ & Lychnis flos-cuculi & & Scotland & $\mathrm{d}$ \\
\hline UWO26-1 & $a_{1}$ & Dianthus carthusianorum & DC-1 & France & $\mathrm{d}$ \\
\hline UWO26-2 & $a_{2}$ & D. carthusianorum & DC-1 & France & d \\
\hline S-4-1 & $a_{1}$ & S. acaulis & & Canada & $\mathrm{e}$ \\
\hline S-4-2 & $a_{2}$ & S. acaulis & & Canada & $\mathrm{e}$ \\
\hline WR31C-2 & $a_{1}$ & S. virginica & & North Carolina (USA) & $\mathrm{e}$ \\
\hline WR31C-1 & $a_{2}$ & S. virginica & & North Carolina (USA) & $\mathrm{e}$ \\
\hline Ger 2-2-1 & $a_{1}$ & M. rubrum & & Germany & $\mathrm{e}$ \\
\hline Ger 2-2-2 & $a_{2}$ & M. rubrum & & Germany & $\mathrm{e}$ \\
\hline
\end{tabular}

${ }^{\mathrm{z}} \mathrm{a}=$ E. D. Garber, University of Chicago; b = P. Oudemans, Rutgers University; c = ATCC, American Type Culture Collection; d = A. Castle, Brock University; $\mathrm{e}=\mathrm{H}$. Alexander, University of Kansas. 
WR 31C-1

WR $31 \mathrm{C}-2$

S-4-2

S-4-1

UWO2 6-2

UWO2 6-1

SIX

NINE

UWO14

1. $\mathrm{C} 436 \mathrm{Y}$

UWO2

2.8.11.81

22001

22000

Ger 2-2-1

Ger 2-2-2

UWO11-1
GTCAGTCCGC GGCACTGGGT GAgGAAgGgC ATCGATGTGG CGAGAgAGGA TAACAAGTAA

61

WR $31 \mathrm{C}-1$

WR $31 \mathrm{C}-2$

$S-4-2$

$S-4-1$

UWO2 6-2

UWO2 6-1

SIX

NINE

UWO14

1.C436y

UWO2

2.8.11.81

22001

22000

Ger 2-2-1

Ger 2-2-2

UWO11-1
AAACGGTCTA
. . . . . .
GTGGCTAAA.
GTGGCTAAA.
--GGCTAAAG
--GGCTAAAG
--GGCTAAA.
--GGCTAAA
--GGCTAAA.
--GGCTAAA
--GGCTAAA.
--GGCTAAA.
--GGCTAAA.
--GGCTAAA.
- -GGCTAAA.
--GGCTAAA.
--GGCTAAAG $\ldots \ldots .$.

....... CGTAC.TT. AG..G..C--

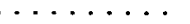

$\ldots \ldots \ldots \ldots$

$\ldots . . . \mathrm{A} . . \mathrm{T} \ldots \mathrm{T}^{-}$. TG.....-

CG..... T GCGAG...AG . G.AG.A...

...... ACGAG. . AT G..G..AG. .

$\ldots . .$. ........-. TG....- ...... ACGAG. . AT G..G..AG..

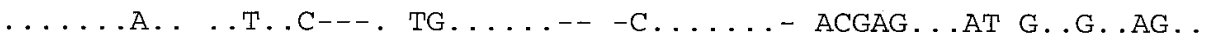

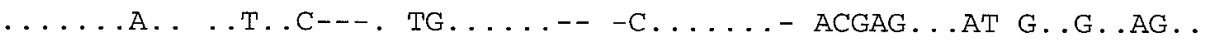

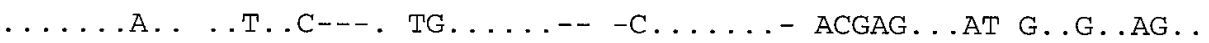

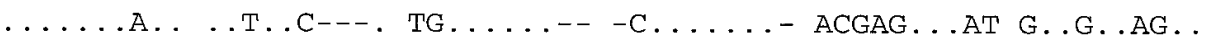

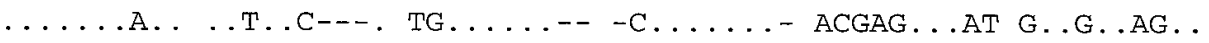

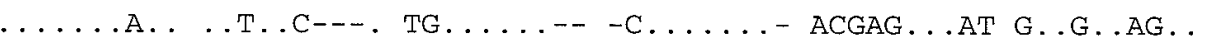

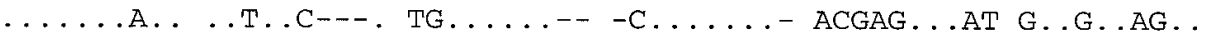

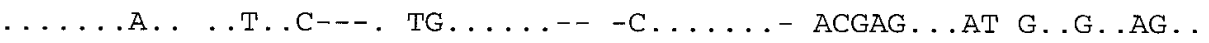

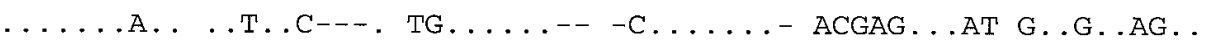

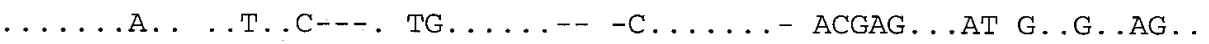

$\ldots . .$. ....T. .C- - TG.....-....- ACGAG..AT G. GG.AG..

121

WR31C-1
WR31C-2
S-4-2
S-4-1
UWO2 6-2
UWO2 6-1
SIX
NINE
UWO14
1. C436y
UWO2
2.8 .11 .81
22001
22000
Ger $2-2-1$
Ger $2-2-2$
UWO11-1

GTCAGCTTCG

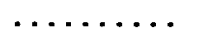

$\ldots \mathbf{G} \ldots \ldots$

$\ldots \mathbf{G} \ldots . .$.

$\ldots \ldots . .$.

$\ldots \ldots \ldots$

$\ldots \ldots . .$.

$\ldots \ldots \ldots$

$\ldots \ldots \ldots$

$\ldots \ldots \ldots$

$\ldots \ldots \ldots$

$\ldots \ldots \ldots \ldots$

$\ldots \ldots \ldots \ldots$

$\cdots$
$\cdots \ldots \ldots$
$\cdots$

$\ldots \ldots \ldots$
CCTTCTTGTC

...................

A.GT.AAA. ACGGTT.CCT

.A.GT.AAA. ACGGTT.CCT

.A. GTCGA. A

- A. GTCGA. A

.A. GTCGA. -

. A. GTCGA. .

. A. GTCGA.

. A. GTCGA. .

.A. GTCGA. .

. A. GTCGA. .

. A. GTCGA. .

. A. GTCGA .

. A. GTCGA. .

. A. GTCGA. .

. A. GTCGA. .
.G.A. .

G.A. .

- - .G.A.T

--- G.A.T

--- G.A.T

- . G.A.T

- - .G.A.T

- . G.A.T

---.G.A.T

--- G.A.T

---.G.A.T

--- .G.A.T

-- . G.A.T
СTCTCCTCCT TAGTÁGGTCT CCACGGTCAT $\cdots \cdots \cdots \cdots$

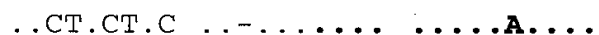
$\ldots$. . ТСТ .. . T- ..... . . ..... $\ldots$. . . . . . . .... . . ..... ... T. . . . T ...... . ...... ... T. ... . T- ..... . T..... ... T. . . . T- ..... . T..... ... Т. . . . T- .... . . ..... ... T.C... . . ..... . ..... $\ldots$...... . T-..... . . .... ... T. . . . T- .... . . .... $\ldots$. . . . . T ..... . . . . . $\ldots$. T. . . . T ..... . . ..... $\ldots$. . . . . Т ..... . . ..... $\ldots$... ... . T-..... . ..... 
Fig. 1. continued from previous page

181

WR31C-1
WR31C-2
S-4-2
S-4-1
UW026-2
UWO26-1
SIX
NINE
UWO14
1.C436y
UWO2
2.8 .11 .81
22001
22000
Ger $2-2-1$
Ger $2-2-2$
UWO11-1

GATCGCTTCC

...........

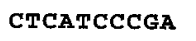

$\ldots \ldots \ldots$

$\ldots \ldots . . .$.

... . . . .

... T. C...

$\ldots \ldots . . .$.

$\ldots \ldots \ldots$

$\ldots \ldots \ldots$

$\ldots \ldots \ldots$.

$\ldots \ldots \ldots$

$\ldots \ldots \ldots$

$\ldots \ldots \ldots \ldots$

$\ldots \ldots \ldots$

........

CCCCTCGgTG

TCACTTCCTC

ACGACTTCTT

ACACCCCCTT

$\ldots \ldots \ldots$

$\ldots \ldots . . .$.

$\ldots \ldots . . .$.

........

$\ldots \ldots \ldots$

$\ldots \ldots \ldots \ldots$

, .

$\ldots \ldots$

$\ldots \ldots \ldots$

$\ldots \ldots \ldots$

$\ldots \ldots \ldots$

$\ldots \ldots \ldots$

$\ldots \ldots \ldots$.

$\ldots \ldots \ldots$

$\ldots \ldots \ldots$

$\ldots \ldots \ldots$

$\ldots \ldots \ldots \ldots, \ldots \ldots \ldots$

$\ldots \ldots \ldots$

$\ldots \ldots \ldots$

$\ldots \ldots \ldots \ldots$

$\ldots \ldots \ldots$

$\ldots \ldots \ldots$

$\ldots \ldots \ldots$

........

$\ldots \ldots \ldots \ldots, \ldots \ldots \ldots$

$\ldots \ldots \ldots$

$\ldots \ldots \ldots$

$\ldots \ldots \ldots$

$\ldots \ldots \ldots$

$\ldots \ldots \ldots$

$\ldots \ldots \ldots$

$\ldots \ldots \ldots$

$\ldots \ldots \ldots$

$\ldots \ldots \ldots \ldots$

$\ldots \ldots \ldots$.

$\ldots \ldots \ldots$

$\ldots \ldots \ldots$

$\ldots \ldots \ldots$

$\ldots \ldots \ldots$

..........

$\ldots \ldots$

.........

$\ldots \ldots$

$\ldots \ldots \ldots$

$\ldots \ldots \ldots$

$\ldots \ldots \ldots$

$\ldots \ldots \ldots$

$\ldots \ldots \ldots \ldots \ldots$

$\ldots \ldots \ldots$

$\ldots \ldots \ldots$

$\ldots \ldots \ldots \ldots \ldots$

$\ldots \ldots \ldots$

$\ldots \ldots$

$\ldots \ldots \ldots \ldots$

$\ldots \ldots \ldots$

$\ldots \ldots \ldots$

$\ldots \ldots \ldots$

WR3 1C -1

WR3 1C-2

S-4-2

S-4-1

UWO2 $6-2$

UWO2 $6-1$

SIX

NINE

UWO14

1. $\mathrm{C} 436 \mathrm{y}$

UWO2

2.8.11.81

22001

22000

Ger 2-2-1

Ger 2-2-2

UWO11-1

241

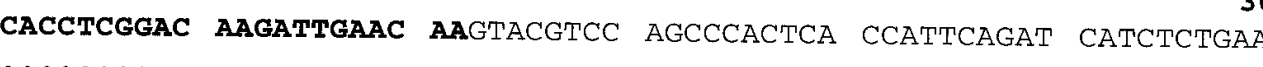

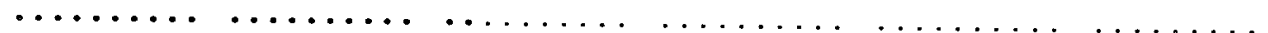

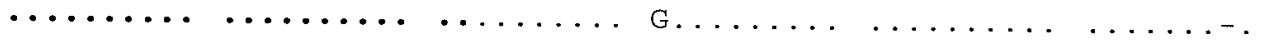

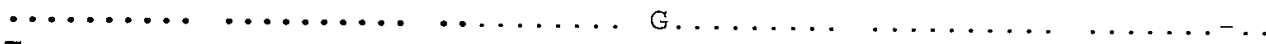

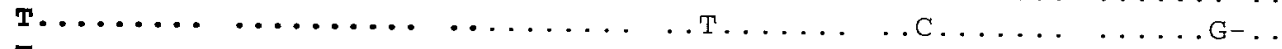

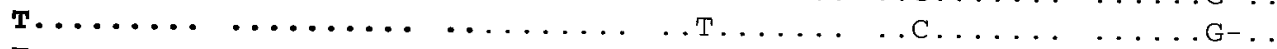

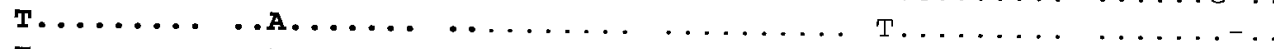

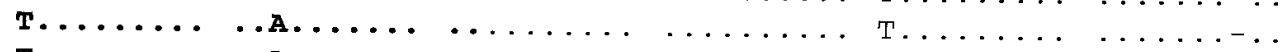

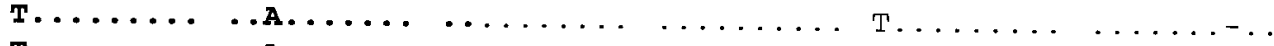

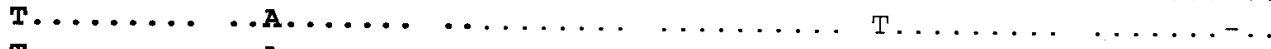

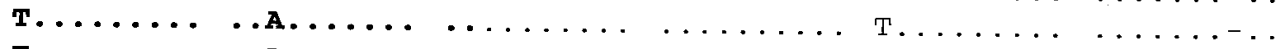

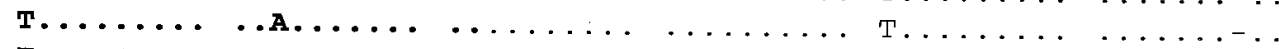

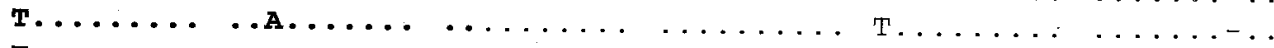

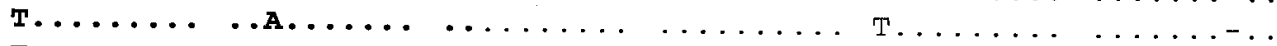

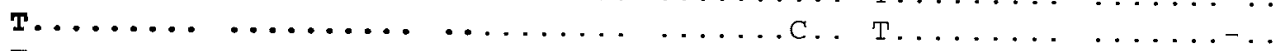

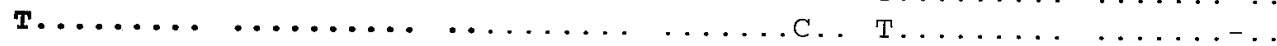

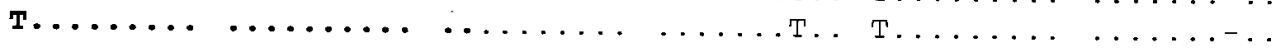

301

WR31C-1 TGCTGACCGC CTCCTCTTCT CGCTCACATC ACAG

WR 31C-2

S-4-2

$S-4-1$

UWO2 6-2

UWO2 6-1

SIX

NINE

UWO14

1. $\mathrm{C} 436 \mathrm{Y}$

UWO2

2.8.11.81

22001

22000

Ger 2-2-1

Ger 2-2-2

UWO11-1

$\ldots \ldots \ldots$

$\ldots \ldots \ldots$ A $\ldots$. . . . TC . . . . .

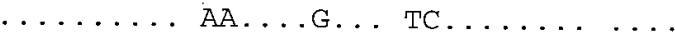

$\ldots \ldots \ldots$. ...... . . ..... . .

$\ldots \ldots \ldots$ A...... TC...... . . .

$\ldots$. . TG. A....G. . TT.A..... G.

$\ldots$. . TG. A...... . T T.A..... G..

$\ldots \ldots$ TG. A....... T TT.A.... G..

$\ldots$.... TG. A....G. . TT.A..... G..

....TG.. A....... TT.A..... G..

$\ldots$. TG. A....... T TT.A.... G..

$\ldots . . \mathrm{TG} .$. A....... TT.A..... G..

$\ldots$...TG. A...... . TT.A..... . .

$\ldots$. . TG. A...... . TT.A..... . .

..... TG. A....... TT.А..... G..

$\ldots$. TG. A....... TC.C.... G. . 
no natural hybridization or introgression between isolates of the fungus found on one species with isolates from the other (3). Moreover, outcrossing between different sources of infective $M$. violaceum appears to be infrequent (4). The modes of transmission and reproduction described above could produce effective reproductive isolation and possibly lead to coevolution, as particular fungal strains become better adapted to their respective host species.

In the present study, we have sought to make within-species comparisons for isolates of $M$. violaceum from different host species by analyzing intron sequences for each isolate. Comparisons of intron sequence diversity have been used to analyze relationships between species. Slade et al. (27) compared closely related pinniped species by constructing multiple nucleargene phylogenies using an approach similar to the one described in this report. In their study, comparison was made with introns and exons for several different genes and the results were compared with phylogenies derived from a mitochondrial region. The authors concluded that each short region of a single-copy nuclear gene alone failed to provide robust phylogenies. In some reported studies of introns within the same species, no differences were reported between individuals. For example, Dorit et al. (10) discovered no sequence variation in a 729-bp intron on the $\mathrm{Y}$ chromosome, in a worldwide sample of 38 human males. Other reports have described discrete types of polymorphisms, including those involving a single nucleotide (5), a specific insertion/deletion $(19,22)$, and a repeated microsatellite (14). In the relatively few reports on fungi, within-species comparisons revealed differences in the presence or absence of specific introns, as well as length and substitution polymorphisms $(6,26,30)$. In fact, one study (30) found that the distribution of three group I introns in Gaeumannomyces graminis was apparently correlated with host specificity of the $G$. graminis varieties. The $\gamma$-tubulin- encoding gene was chosen for analysis in the present study because the protein has been demonstrated to be highly conserved in amino acid sequence across all kingdoms (16; unpublished data). Thus, selective constraints at the amino acid level would be expected to limit the degree of within-species variation in coding regions for this gene to synonymous positions; no similar constraints should have been placed on intron sequences. In contrast to previous examinations of introns for population studies $(5,14,19,22)$, the data in the present study show several different types of dispersed polymorphisms, both base substitutions and insertion/deletions. These polymorphisms suggest strongly that $M$. violaceum formae speciales from divergent hosts have been reproductively isolated long enough to have acquired changes within the gene encoding the highlyconserved $\gamma$-tubulin protein. Moreover, such differences may serve as a diagnostic means of identifying isolates of a particular forma specialis.

Although the phylogeny of plants in the Caryophyllaceae is still problematic, for hosts in this study the following relationships appear to hold $(8,9,20)$ : Of the eight host species from which $M$. violaceum isolates in this study were obtained, all are within the subfamily Silenoideae. While Dianthus is clearly outside the tribe Sileneae, Lychnis and Melandrium are included. Based on ITS sequences, Desfeux and Lejeune (8) have grouped Lychnis, Melandrium, and Silene into the single genus, Silene. Dianthus is a closely related genus that shares the characteristic of dioecy with some of the other hosts (e.g., $S$. latifolia and $S$. dioica) (8). M. rubrum and $S$. latifolia are appear quite close phylogenetically, as evidenced by previous classifications of S. latifolia as M. latifolia (9). Although $S$. latifolia and $S$. acaulis are both in Silene, they are in different clades within this genus (8). Moreover, unlike $S$. latifolia, some subspecies of $S$. acaulis display trioecy or gynodioecy (8). S. vir-

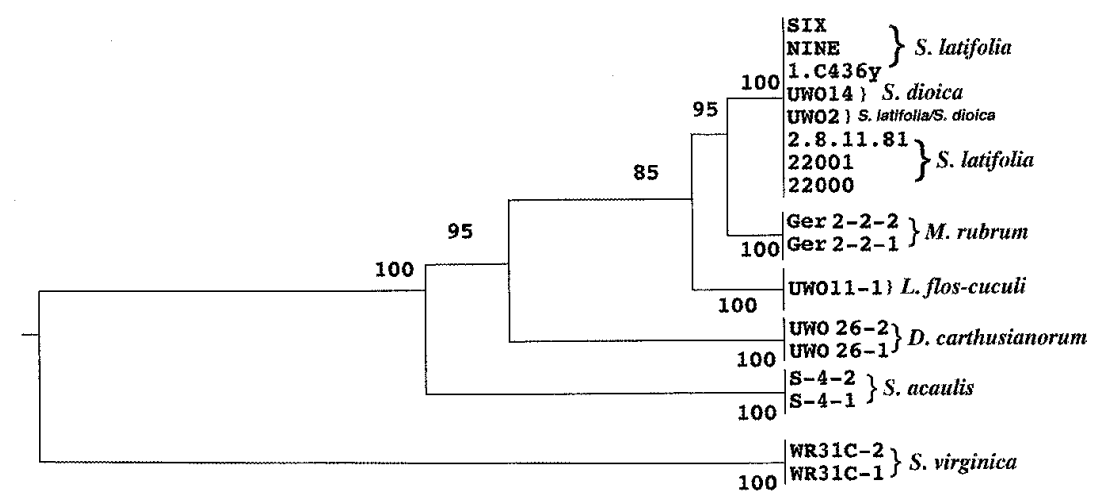

Fig. 2. Bootstrap analysis of $\gamma$-tubulin gene regions. DNA sequences of the sixth intron, seventh exon, and seventh intron for the Mycobotryum violaceum $\gamma$-tubulin gene were compared for each isolate using, PAUP 3.1.1. This single parsimonious tree was produced from 100 replications, using the branch-and-bound search algorithm; this is a 50\% majority rule consensus tree. Host species of origin are indicated after each strain. ginica, the only host species in this study native exclusively to North America, appears to be quite distinct phyogenetically from the other hosts originating in Europe (3).

The region of the $\gamma$-tubulin gene examined in this study was chosen primarily for convenience. When subsections of this region (e.g., the sixth intron alone) were compared, not all pathovars were clearly resolved. For example, in analyses of intron 6 alone, the isolates from M. rubrum were included with the $S$. latifolia clade. These results reflect the probable phylogenetic similarity of the respective host species for these isolates (8). They are also consistent with similar electrophoretic karyotypes for the M. rubrum isolates relative to those from S. latifolia (23). Moreover, in analyses of the combined region (intron 6, exon 7, and intron 7), the $M$. rubrum isolates formed a sister group to the isolates from S. latifolia.

In those instances where isolates of different formae speciales remained indistinguishable (i.e., strain UWO2, from a $S$. latifolia/S. dioica hybrid host, and strain UWO14, from $S$. dioica, consistently grouped with the isolates from $S$. latifolia), it is possible that analysis of additional unexamined introns and exons might permit separation of such isolates. Alternatively, the inability to distinguish some isolates may mean that the strains in question all belong to the same forma specialis and share the same host range. Strain 1.C436y, for example, was originally isolated from $S$. dioica, but its host range includes $S$. latifolia as well. Such findings are consistent with the observations that field collections of $M$. violaceum from $S$. dioica or from $S$. latifolia/S. dioica hybrid hosts were pathogenic on S. latifolia (11). Further, isolates from $D$. deltoides, $L$. floscuculi (11), or S. virginica (3) failed to cause disease on S. latifolia or did so very poorly. In natural infections, such restricted host range might have developed due to different modes of pathogen transmission; for example, vegetative transmission appears to be the predominant mode of disease spread in $S$. virginica, whereas for $S$. latifolia transmission more often occurs via insect pollinators or wind (3). Of particular interest is the fact that analyses of the combined DNA region found the isolates from $S$. virginica to be most distant from the remaining strains in this study. These findings are consistent with the degree of polymorphism observed in electrophoretic karyotype for these isolates relative to those of the others (23). Since isolates WR31C-1 and WR31C-2 came from the only host species ( $S$. virginica) in this study known to be exclusively native to North America (32), it is tempting to speculate that reproductive isolation has played a major role in distinguishing these isolates from those introduced relatively recently from Europe. It should be empha- 
sized that anther smut on S. latifolia showed no apparent geographic pattern in terms of the region sequenced and they were indistinguishable in DNA sequence from isolates obtained from $S$. dioica (another European native). Thus, smut on S. latifolia in the United States is probably due to a migration event from Europe, and not to a host shift from a native Caryophyllaceae host. That the relationships within and among pathovars in this study were established with a relatively small sample size suggests that polymorphisms in the $\gamma$-tubulin gene among M. violaceum pathovars are widespread and that these polymorphisms might parallel those of host phylogenies or reproductive similarities in a much larger sampling. Ideally, the correlation of intron polymorphisms with host range can be further extended to serve as a powerful diagnostic tool in examining pathogenesis of this and other fungal plant pathogens.

\section{ACKNOWLEDGMENTS}

The advice and helpful comments of Jon Ahlquist and Richard Harrison in previewing the manuscript are greatly appreciated as are the comments of two anonymous reviewers. Pete Thrall and Janis Antonovics provided information about the phylogenetic relationships of host species. This work was supported in part by an Arts and Sciences Research Grant and by a President's Research Initiative Grant from the University of Louisville.

\section{LITERATURE CITED}

1. Alexander, H. 1987. Pollinator limitation in a population of Silene virginica infected by the anther-smut fungus Ustilago violacea. J. Ecol. 75:771-780.

2. Antonovics, J. 1992. Towards community genetics. Pages 426-449 in: Ecology and Evolution of Plant Resistance. R. S. Fritz and E. L. Simms, eds. University of Chicago Press, Chicago.

3. Antonovics, J., Stratton, D., Thrall, P. H., and Jarosz, A. M. 1996. The anther-smut disease (Ustilago violacea) of fire-pink (Silene virginica): Its biology and relationship to the anther-smut disease of white campion (Silene alba). Am. Midl. Nat. 135:130-143.

4. Baird, M. L., and Garber, E. D. 1979. Genetics of Ustilago violacea. V. outcrossing and selfing in teliospore inocula. Bot. Gaz. 140:89-93

5. Braun, A., Ambach, H., Bichlmeier, R., Kammerer, S. and Roscher, A. A. 1995. Population study of a sequence polymorphism in intron 2 of the human beta-globin gene. Hum. Genet. 95:352.
6. Chen, F., Currie, B. P., Chen, L. C., Spitzer, S. G., Spitzer, E. D., and Casadevell, A. 1995. Genetic relatedness of Cryptococcus neoformans clinical isolates grouped with repetitive DNA probe CNRE-1. J. Clin. Microbiol. 33:2818-2822.

7. Deml, G., and Oberwinkler, F. 1982. Studies in heterobasidiomycetes. Part 24. On Ustilago violacea (Pers.) Rouss. from Saponaria officinalis. L. Phytopathol. Z. 104:345-356.

8. Desfeux, C., and Lejeune, B. 1996. Systematics of Euromediterranean Silene (Caryophyllaceae): Evidence from a phylogenetic analysis using ITS sequences. C. R. Acad. Sci. Ser. 3 319:351-358.

9. Desfeux, C., Maurice, S., Henry, J.-P., Lejeune, B., and Gouyon, P.-H. 1996. Evolution of reproductive systems in the genus Silene. Proc. Roy. Soc. London Ser. B 263:409-414.

10. Dorit, R. L., Akashi, H., and Gilbert, W. 1995. Absence of polymorphism at the ZFY locus on the human $\mathrm{Y}$ chromosome. Science 268:1183-1185.

11. Garber, E. D., Baird, M. L., and Weiss, L. M. 1978. Genetics of Ustilago violacea. II. polymorphism of color and nutritional requirements of sporidia from natural populations. Bot. Gaz. 139:261-265.

12. Garber, E. D., and Day, A. W. 1985. Genetic mapping of a phytopathogenic basidiomycete, Ustilago violacea. Bot. Gaz. 146:449459.

13. Jukes, T. H., and Cantor C. R. 1969. Evolution of protein molecules. Pages 21-132 in: Mammalian protein metabolism. H. N. Munro, ed. Academic Press, New York.

14. Leveziel, H., Rodellar, C., Leroux, C., Pepin, L., Grohs, C., Vaiman, D., Mahe, M. F., Martin, P., and Grosclaude, F. 1994. A microsatellite within the bovine kappa-casein gene reveals a polymorphism correlating strongly with polymorphisms previously described at the protein as well as the DNA level. Anim. Genet. 25:223-228.

15. Lin, S. R., Chang, S. C., Lee, C. C., Shen, M. C., and Lin, S. W. 1995. Genetic diagnosis of haemophilia A of Chinese origin. Br. J. Haematol. 91:722-727.

16. Logsdon, J. 1995. Late origin of introns in tubulin genes. Ch. 4 in: Origin and evolution of spliceosome introns. Ph.D. diss., Indiana University, Bloomington, IN.

17. Luo, H., and Perlin M. H. 1993. The $\gamma$ tubulin-encoding gene of the basidiomycete fungus, Ustilago violacea, has a long 5'untranslated region. Gene 137:187-194.

18. Marez, D., Sabbagh N., Legrand, M., LoGuidice, J. M., Boone, P., and Broly F. 1995. A novel CYP2D6 allele with an abolished splice recognition site associated with the poor metabolizer phenotype. Pharmacogenetics 5:305-311.

19. Nakai, K., Itoh, C., Miura, Y., Hotta, K., Musha, T., Itoh, T., Miyakawa, T. , Iwasaki, R. and Hiramori, K. 1994. Deletion polymorphism of the angiotensis I-converting enzyme gene is associated with serum ACE concentration and increased risk for CAD in the Japanese. Circulation 90:2199-2202.

20. Oxelman, B., and Liden, M. 1995. Generic boundaries in the tribe Sileneae (Caryophyllaceae) as inferred from nuclear rDNA sequences. Taxon 44:525-542.

21. Palumbi, S. R., and Baker, C. S. 1994. Contrasting population structure from nuclear intron sequences and mtDNA of humpback whales. Mol. Biol. Evol. 11:426-435.

22. Peller, S., Kopilova, Y., Slutsky, S., Halevy, A., Kvitko, K., and Rotter, V. 1995. A novel polymorphism in intron 6 of the human p53 gene: A possible association with cancer predisposition and susceptibility. DNA Cell Biol. 14:983-990.

23. Perlin, M. H. 1996. Pathovars or formae speciales of Microbotryum violaceum differ in electrophoretic karyotype. Int. J. Plant Sci. 157:447-452

24. Sanger, F., Nicklen, S., and Coulsen, A. R. 1977. DNA sequencing with chainterminating inhibitors. Proc. Nat. Acad. Sci. USA 74:5463-5467.

25. Savile, D. B. O. 1954. The fungi as aids in the taxonomy of the flowering plants. Science 120:583-585.

26. Shinohara, M. L., Lobuglio, K. F., and Rogers, S. O. 1996. Group-I intron family in the nuclear ribosomal RNA small subunit genes of Cenococcum geophilum isolates. Curr. Genet. 29:377-387.

27. Slade, R. W., Moritz, C., and Heideman, A. 1994. Multiple nuclear-gene phylogenies: application to pinnipeds and comparison with a mitochondrial DNA phylogeny. Mol. Biol. Evol. 11:341-356.

28. Spencer, J. L., and White, H. E. 1950. Anther smut of carnation. Mass. Agric. Exp. Stn. Contrib. 751:291-299.

29. Tamura, K., and Nei, M. 1993. Estimation of the number of nucleotide substitutions in the control region of mitochondrial DNA in humans and chimpanzees. Mol. Biol. Evol. 10:512-526.

30. Tan, M. K., and Wong, P. T. W. 1996. Group I introns in 26S rRNA genes of Gaeumannomyces graminis as possible indicators of host specificity of $G$. graminis varieties. Mycol. Res. 100:337-342.

31. Thompson, J. D., Higgins, D. G., and Gibson, T. J. 1995. Clustal W: improving the sensitivity of progressive multiple sequence alignment through sequence weighting, position gap penalties and weight matrix choice. Nucl. Acids Res. 22:4673-4680.

32. Thrall, P. H., Biere, A., and Antonovics, J. 1993. Plant life history and disease susceptibility-The occurrence of Ustilago violacea on different species within the Caryophyllaceae. J. Ecol. 81:489-498.

33. Zillig, H. 1921. Über spezialiserte formen beim antherenbrand Ustilago violacea (Pers. ) Fuckel. Zentralbl. Bakteriol. Parasitenkd. 53:33-73. 